\title{
L'appropriation des Technologies de l'information et de la communication pour remédier au malaise de notre capitale
}

\author{
Lahcen Darhouani \\ Consultant en Technologies de l'information \\ et développement régional
}

$\int$ a mondialisation des marchés et des enjeux socio-économiques entraîne la « compétition des territoires » et donne lieu à une nouvelle hiérarchie territoriale. Pour toutes sortes de raisons, les territoires n'ont pas les mêmes forces ni les mêmes faiblesses, ce qui indique d'emblée aux planificateurs certaines avenues prioritaires pour le développement : savoir profiter des points forts et faire le nécessaire pour corriger les faiblesses. Au Québec, un consensus semble s'établir parmi nos élites: voir Montréal occuper une place de premier plan au sein des « régions urbaines mondiales ». Conformément à cette vision, le gouvernement a souvent réservé, par exemple dans la mise en place des différents fonds de développement ou dans son récent budget, la part du lion à la métropole. En fait, les décideurs politiques agissent avec le sentiment plus ou moins avoué que les zones économiques du Québec doivent soutenir la métropole dans son effort pour s'imposer sur l'échiquier mondial. Certes, il faut souhaiter que Montréal demeure une puissante locomotive économique. Mais plusieurs sont amenés à penser que les territoires hors métropole comptent pour peu dans les stratégies de développement du Québec et que, en particulier son second pôle économique, la capitale, semble être oublié.

Cet article porte principalement sur la ville de Québec et son rôle comme capitale, mais ce faisant on ne peut évidemment pas faire abstraction de son entourage. Ces derniers temps, dans le prolongement du questionnement des médias sur «l'énigme de Québec", divers analystes se sont intéressés aux conditions so- ciales et politiques propres à la région de Québec. Mais on n'a guère tenu compte des facteurs économiques pouvant contribuer à expliquer le sentiment d'impuissance, le mécontentement, et le désir du changement de la population de la ville et des régions administratives qui l'environnent: capitale nationale et Chaudière-Appalaches. Le texte qui suit procède de l'observation et de la réflexion d'un simple citoyen, qui a eu le privilège d'habiter à Québec et qui s'est intéressé de près, depuis une dizaine d'années et comme spécialiste du développement régional, au fonctionnement de l'activité économique dans la région de Québec.

\section{Une concertation difficile entre deux régions}

Se faisant face de part et d'autre du fleuve, Québec et Chaudière-Appalaches affichent, quant à leur développement, des visions divergentes, sources de confusion, freins à une action commune et, en certains cas, à une stimulante compétition. Selon l'ancien maire de Lévis, Jean Garon, « on a beaucoup trop peur de la compétition dans notre coin des Amériques. Les Québécois pensent toujours à s'entendre entre eux pour faire des "réseaux"... On ne se compétitionne jamais et on s'imagine qu' on va être les champions à l'international. Voyons donc! Ici, on attend toujours que le gouvernement mette de l'argent... comme un oiseau dans le nid, qui attend, le bec ouvert, que la mère mette quelque chose dedans ". Cette sortie visant les pratiques des acteurs les plus influents de la région de 
Québec témoigne d'un certain état d'esprit qui sévit dans Chaudière-Appalaches.

En effet, les acteurs de la rive sud laissent voir un certain malaise lorsqu'il s'agit de coordonner leurs actions avec leurs homologues de la rive nord. Ils notent que ces derniers mettent généralement de l'avant des projets d'envergure, dont les retombées seront souvent plus importantes dans la capitale. Ils estiment que l'administration de Québec, en œuvrant à la mise en place de structures institutionnelles, a cherché à aligner le développement des deux régions autour de sa propre stratégie, laquelle favorisait de grands projets impliquant nécessairement l'appui financier des gouvernements supérieurs. C'est ainsi que sont perçues les pratiques de l'ancienne direction et des acteurs économiques de la ville de Québec. Lors du dernier forum économique, on a remarqué que les gens d'affaires se sont entendus pour appuyer plusieurs grands projets de développement, dont le port méthanier Rabaska, les Fêtes du $400^{\mathrm{e}}$, la rénovation de l'aéroport Jean-Lesage, le Centre de prévention de l'obésité de l'hôpital Laval et le sauvetage du zoo. Quant au ministre responsable de la région, les dossiers prioritaires pour lui sont l'aéroport, les fêtes du $400^{\mathrm{e}}$, l'agrandissement du centre de foires et le projet du massif de Petite-Rivière-Saint-François.

Tout souhaitables que paraissent ces projets, il semble que la plus grande part des retombées économiques ira à la rive nord, et certains acteurs de la rive sud ne sont pas loin d'y voir une manœuvre qui les empêcherait de décider eux-mêmes de leur développement, tout en donnant aux acteurs de la capitale la visibilité recherchée. En somme, le souhait d'associer les deux régions est perçu par plusieurs, au sud, comme une stratégie qui permettrait à ceux du nord de contrôler le développement des deux régions.

Dans la région de la Capitale, la vision est différente. Québec et ses environs profitent naturellement de la présence du siège du gouvernement et du statut de capitale pour tisser des liens à l'échelle nationale et internationale, à l'appui des stratégies de développement mises de l'avant par les principaux acteurs économiques et politiques. Il apparaît, par ailleurs, à ces acteurs que le développement des deux régions passe par une forme de complémentarité. Selon l'ancien maire de Québec, Jean-Paul L'Allier, «il faut chercher les complémentarités si l'on veut se développer.
On n'a pas suffisamment de force et de volume, sur le plan des paramètres du développement moderne, pour affirmer que Lévis, avec ses 125000 habitants, peut faire concurrence à Chicago, Boston, etc. On a intérêt non pas à s'assimiler les uns les autres, mais à chercher nos complémentarités ». En fait, « les cours d'eau divisent les villes et ce, non seulement sur le plan physique : c'est la même chose à Hull-Ottawa, à Budapest et ailleurs, dans les municipalités qui sont partagées par un fleuve ou par une rivière. Ça devrait les unir mais, traditionnellement, on perçoit cela comme une opposition $»$.

L'ancien maire de Lévis n'est pas d'accord : « Dans la rive sud, on est conscient de cette différence. Québec et Chaudière-Appalaches ne voient pas ça de la même façon. À Québec, ils voient ça de façon institutionnelle. C'est pour ça que je ne voulais pas qu'on le fasse ensemble. [On] m'a déjà dit que [le maire de Québec] et moi étions différents : alors que lui voulait développer avec des fonds publics, moi je voulais le faire avec l'entreprise ». Il s'agit donc ici non seulement d'une certaine opposition nord-sud, mais d'une remise en question du « modèle québécois » puisque c'est de la capitale nationale qu'il s'agit et de son rôle par rapport à son proche environnement et au reste du Québec.

Il est vrai qu'une réelle complémentarité entre les deux régions augmenterait leur compétitivité, si l'on considère par exemple que le prolongement prévu de l'autoroute 73 vers Saint-Georges, et éventuellement vers les États-Unis, permettra aux entreprises des deux régions d'accéder à un marché énorme. Les entreprises des deux régions peuvent s'associer, former des réseaux, établir des partenariats, etc. Mais on peut en même temps soutenir que, à l'heure des Technologies de l'information et de la communication (TIC), rien ne les empêche d'établir également de telles relations avec d'autres entreprises implantées n'importe où, indépendamment de la proximité géographique.

\section{Lors du dernier forum économique, on a remarqué que les gens d'affaires se sont entendus pour appuyer plusieurs grands projets de développement.}

Il n'y a pas lieu de trancher ici entre deux visions divergentes, avançant chacune ses propres arguments. 
Éventuellement, il serait sûrement intéressant d'examiner plus à fond cette question : pourquoi les acteurs de la capitale insistent-ils pour s'associer aux gens de la rive sud, qui ne cachent pourtant pas leur peu d'intérêt? Et pourquoi ceux de la rive sud sont-ils réticents devant les propositions de Québec ? Pour l'observateur attentif, il semble que le nœud du problème tienne au rôle et aux dynamiques propres à la capitale, dont les objectifs ne peuvent pas toujours coïncider avec ceux des territoires environnants. Il convient néanmoins d'esquisser les éléments d'une stratégie qui devrait permettre aux collectivités des deux régions de se prendre réellement en charge. Mais, pour l'instant, examinons la situation dans la capitale.

\section{Des vues divergentes à Québec}

C'est avec curiosité et étonnement qu'on observe les forces en présence et leur comportement. Les dynamiques économiques de la capitale ont été depuis longtemps influencées par la présence de réseaux forts, articulés principalement autour de dirigeants de la Ville et de la Commission de la Capitale nationale. Ces réseaux et les groupes de pression, qui se forment au gré des dossiers qu'on veut faire avancer, ont eu souvent des effets limitatifs sinon regrettables sur le cours de l'activité économique. Il semble, par exemple, que des entrepreneurs désireux d'investir dans la région doivent, s'ils veulent mener leurs plans à bien, obtenir l'aval de ces réseaux, où les acteurs majeurs choisissent les projets qu'ils désirent voir avancer et arrivent à en imposer certains, malgré parfois 1'opposition des populations locales.

Le fonctionnement de ces réseaux est présentement remis en question par la nouvelle administration municipale de Québec ainsi que par le gouvernement provincial, ce qui modifie la donne et crée un état d'expectative pour les dynamiques économiques de la capitale. Le fonctionnement du conseil municipal de Québec en témoigne : l'opposition actuelle y insiste sur la nécessité de «se préoccuper du développement», de «maintenir l'élan » donné par l'ancien conseil, alors que la nouvelle administration préfère concentrer ses efforts d'abord sur la gestion interne de la Ville. Elle prévoit en effet consacrer près des deux tiers de son programme triennal d'immobilisation à l'amélioration des réseaux d'aqueduc, d'égout, à l'état des bâtiments de la Ville et au pavage des rues. Les héritiers de l'ancienne équipe décrivent la nouvelle orientation comme s'opposant au développe- ment de la ville et de la région, et soutiennent que les choix de l'ancienne administration pouvaient garantir ce développement.

Depuis l'arrivée de la nouvelle équipe à la mairie de Québec, la ville semble donc "coupée en deux». L'ancienne administration avait articulé sa planification autour de deux axes : la dimension internationale, qui incitait la Ville à s'ouvrir d'avantage sur les pays francophones, notamment sur la France; et l'embellissement de la capitale. L'ancienne administration a souvent eu l'appui inconditionnel de la Commission de la Capitale nationale. Les efforts de l'équipe dirigeante étaient consacrés à la création d'une capitale forte, pouvant se comparer aux autres capitales du monde. On a signé des ententes avec les dirigeants d'autres capitales, voire d'autres pays. Au cours du dernier exercice, la ville de Québec avait organisé 44 missions à l'étranger. Ce qui a requis l'envoi à l'extérieur de plusieurs dirigeants et fonctionnaires de la Ville, tantôt pour renforcer les liens «diplomatiques » de la Ville, tantôt pour promouvoir auprès des futurs immigrants la Capitale nationale comme lieu d'établissement. L'équipe dirigeante était ainsi amenée à s'occuper de moins près des affaires quotidiennes de la Ville. Pour compenser, on créait des organismes dédiés aux différentes missions. Cette orientation s'est beaucoup accélérée à la suite des fusions municipales de 2001, avec 1'alourdissement conséquent des structures administratives. Les héritiers de l'ancienne équipe, qui constituent désormais l'opposition au conseil de ville, incarnent cette tendance et souhaitent continuer sur la même lancée.

À l'opposé, l'administration actuelle «n'entend se préoccuper que de gestion interne » de la ville. Certains lui reprochent son désintérêt pour le développement, estimant que mettre la priorité exclusivement sur la gestion interne de la ville est "malsain et dangereux ». Pendant un moment, Claude Larose, ce haut dirigeant du Renouveau municipal de Québec, parti de l'opposition, a envisagé de défier le choix de la population de Québec et a appelé ses troupes à imposer sa vision : "Les gens de Québec ont fait un choix très clair : ils ont fait le choix de mettre la priorité sur la gestion interne de la ville, quitte à mettre en péril l'élan de développement de la ville et de la région. [Mais] si on laisse aller les choses, le laisser-faire va briser l'élan ». Ce sont là deux visions résolument opposées, qui amènent l'administration actuelle de la ville et l'opposition à perdre du temps à « se crêper le 
chignon " plutôt que de faire avancer des dossiers importants. La récente entrevue du président de la Chambre du commerce de Québec, où il critiquait la mairesse et l'actuelle administration de la ville, montre que le milieu des affaires attend avec impatience une orientation et des décisions devant favoriser la performance économique.

Quoi qu'il en soit, il reste quelques questions auxquelles il ne semble pas y avoir de réponses claires. Pour la gestion d'un même territoire, on trouve le conseil municipal de la ville de Québec et aussi la Commission de la Capitale nationale. Et comme l'essentiel de l'appareil gouvernemental s'y trouve également, celui-ci y exerce une influence certaine. En matière notamment de relations internationales, de développement économique et d'immigration, quels sont les champs de compétence de l'un et de l'autre organisme? Les fonctions attribuées à chaque niveau sont-elles délimitées et précisées afin que chacun se contente de jouer le rôle historique qui lui revient, pour éviter les recoupements, la duplication et la dispersion des forces ? C'est en fonction des réponses à ces questions que les collectivités des deux régions concernées doivent juger de la pertinence des interventions d'acteurs de l'un ou l'autre palier décisionnel, en vue de s'assurer d'un développement économique satisfaisant, tout en donnant à Québec la place qui lui revient comme Capitale nationale. C'est sous cet éclairage qu'il faudra définir les relations que Québec, comme centre politique, doit jouer non seulement face à Chaudière-Appalaches, mais aussi par rapport à l'ensemble du territoire québécois.

\section{Pour ce qui est de Québec, une observation attentive laisse voir en effet que, malgré les efforts fournis par les acteurs économiques et politiques depuis deux décennies, les stratégies économiques ne semblent pas conduire au développement espéré.}

\section{Une dynamique économique atteinte de myopie}

Les médias rapportaient récemment les étonnants échanges de politesses entre les dirigeants des deux chambres de commerce de la capitale, notamment entre Pierre Dolbec et André Di Vita : «Ils ont leur opinion et nous [la CCQ] avons la nôtre. Nous, c'est une situation rationnelle de gens d'affaires que nous proposons. Ils [les dirigeants de la CCEQ] peuvent avoir un point de vue différent s'ils le veulent». Il s'agit là d'un exemple des attitudes contre-productives, révélatrices du peu de communication et de la mésentente qui divisent le monde des affaires. Dans ce contexte, comment penser «qu'il est possible de parler de choix collectifs, de vision économique régionale, de convergence, de défis communs, de porteparole unique pour la défense et la représentation du secteur privé, quand deux chambres de commerce se comparent, se chevauchent, utilisent Québec dans leur appellation, sollicitent les mêmes membres et les mêmes partenaires financiers, rencontrent les mêmes chefs de file et prétendent aux mêmes tables de discussion ". Ces vaines rivalités pervertissent les pratiques d'affaires, freinent nos dynamiques économiques et mènent trop souvent à un chauvinisme stérilisant.

Pour ce qui est de Québec, une observation attentive laisse voir en effet que, malgré les efforts fournis par les acteurs économiques et politiques depuis deux décennies, les stratégies économiques ne semblent pas conduire au développement espéré. On se base sur un faible taux de chômage et sur le fait que Québec se situerait au cinquième rang des municipalités canadiennes pour montrer la vitalité économique de la région. Mais est-ce bien la réalité ? Alors que plusieurs de nos jeunes, incapables de trouver du travail ici, quittent la région; que nombre de PME périclitent. De plus, on peine à attirer des immigrants et surtout à les garder, alors que le vieillissement de la population commence à faire sentir ses effets dans la société et dans l'économie.

Parmi notre élite d'affaires, la vision partielle (sinon partiale) évoquée plus haut entraîne la morosité d'une partie de l'économie qui arrive mal à s'adapter aux fluctuations d'un marché mondial mouvant et fortement concurrentiel. À Québec par exemple, Jean-Paul l'Allier a décrit la situation comme suit: "Quand l'économie fait ici des bonds vers le haut, ils sont moins élevés qu'ailleurs. Quand ce sont des creux vers le bas, ils sont moins profonds qu'ailleurs. Il en résulte plus de stabilité dans la région ». Ou peut-être que cette stabilité, due en bonne part à la présence d'une nombreuse fonction publique, occulterait plutôt du sur-place, ce qui semble concorder avec une observation du Conference Board. Leur étude confirme l'existence d'un écart important entre le territoire de Québec, où les principaux indicateurs économiques et 
sociaux donnent l'impression d'une certaine croissance, et l'ensemble de la province, qui traîne péniblement derrière les autres provinces canadiennes.

Les quelques éléments présentés plus haut sont une indication d'une sorte de myopie qui affecte les dynamiques économiques de la région de la Capitale nationale. Les commentaires désabusés, parfois amers, de dirigeants de PME, nous ont plusieurs fois sidérés. Faisant part de leurs expériences, ils se plaignent du peu d'intérêt, de l'incompréhension, voire de l'incompétence de représentants d'organismes ayant pour mission de les accompagner; bref, ils se sont sentis abandonnés. À l'ère de la mondialisation du marché et faute de formation adéquate, les pratiques de ces organismes aboutissent trop souvent à un cul-de-sac. Ces organismes ont proliféré au fil des ans, en même temps que les fonds nécessaires à leur gestion; mais les résultats qu'ils obtiennent sont rarement à la hauteur des besoins de développement de toute la région.

\section{Particulièrement dans la région de la Capitale nationale, il semble manquer aux principaux acteurs des idées porteuses, une vision globale du développement à promouvoir.}

Cela, parmi d'autres facteurs, aide à comprendre pourquoi nos PME se sentent incapables de s'imposer dans le marché international. Tant que durera cette situation, et malgré l'ouverture manifestée dernièrement par certains acteurs publics et parapublics, ce sentiment d'impuissance continuera à freiner les dynamiques économiques de la capitale. Par ailleurs le nombre relativement élevé des organismes "d'aide ", les rencontres et les activités qu'ils organisent, donnent l'impression que l'économie de la région avance. Cette illusion est souvent renforcée par la mise à l'avant-scène d'un nombre limité d'entreprises de taille importante, implantées dans le territoire et qui réussissent bien.

Particulièrement dans la région de la Capitale nationale, il semble manquer aux principaux acteurs des idées porteuses, une vision globale du développement à promouvoir. Ils donnent l'impression de relever d'importants défis, en préférant les grands projets et les manifestations d'envergure, alors qu'il y a dans la réalité des indices d'une sorte d'incohérence entre les exigences d'une stratégie inventive de développement et les pratiques quotidiennes. Ce qui est de nature à attiser la méfiance chez certains acteurs, et à décourager de potentiels investisseurs d'ici et d'ailleurs.

\section{Des PME négligées}

Des vues et des pratiques divergentes, voire opposées, dans le monde des affaires comme chez les élus municipaux, quant à la planification du développement économique, sont sources d'inégalité des chances pour les entrepreneurs. D'un côté, un petit nombre d'entreprises, généralement de taille importante, profitent davantage des structures, financières et autres, mises en place par les acteurs influents et les organismes d'appui au développement. Ces organismes tirent parti d'une aide financière substantielle, offerte principalement par le gouvernement fédéral, par l'intermédiaire de Développement Économique Canada. À ces organismes s'associent généralement d'autres organisations comme la ville de Québec, la Commission de la Capitale nationale, etc. Les représentants du gouvernement provincial, notamment le ministère de du Développement économique, de l'Innovation et de l'Exportation ainsi que Emploi Québec, sont aussi appelés à y participer.

D'un autre côté, on trouve un nombre considérable de PME autour desquelles gravite une grande partie de la population, souvent des jeunes, qui occupent des emplois peu rémunérés : ouvriers et journaliers au salaire minimum, employés à situation précaire (emplois à temps partiel, emplois à contrat, etc.). Or, trop souvent, malgré leur importance, ces PME et leurs employés sont oubliés par des décideurs à court de moyens ou d'imagination pour les intégrer dans le modèle en place.

Ces PME constituent la plate-forme de notre économie régionale. Elles sont souvent encadrées et accompagnées par certains organismes, comme les CLD (Centres locaux de développement), les SADC (Sociétés d'aide au développement des collectivités), etc., dont les représentants sont davantage tournés vers les problèmes locaux que vers les enjeux du marché international. Cette situation paralyse souvent les efforts d'internationalisation des PME de la région. On peut y voir le résultat d'un contexte de médiocrité, qui ne peut que contribuer à l'asphyxie d'initiatives locales. 
On constate trop souvent par ailleurs que les responsables locaux d'organismes ayant pour mission d'aider l'entreprise n'ont pas la formation voulue pour accompagner efficacement les PME au moment où il leur faut s'approprier les outils technologiques et le savoir indispensable à leur survie. Alors, il ne faut pas s'étonner que les PME, comme une partie de la population concernée, se sentent négligées et isolées, soumises aux aléas de forces anonymes qu'elles ne contrôlent pas. Le rôle économique des PME, de même que leur facilité à s'arrimer aux dynamiques de développement de leur milieu, restera limité sans la mise en place de structures d'accompagnement adéquates. Entre les pouvoirs publics et les dirigeants d'entreprises, les organismes d'aide au développement sont des intermédiaires, des ponts parfois éprouvants, trop souvent inefficaces. Une sérieuse remise en question de nos stratégies de développement paraît donc s'imposer.

\section{Repenser nos façons de faire}

Le survol qui précède a mis en lumière, dans les régions de la Capitale nationale et de ChaudièreAppalaches, des attitudes et des orientations souvent défavorables à l'activité économique et à la prospérité qu'on en attend. La complémentarité souhaitée entre nos deux régions ne se concrétise pas. La dynamique économique ambiante, particulièrement à la ville de Québec, a utilement patronné des entreprises et des projets de premier plan, mais n'a guère compris le rôle et l'importance des PME et d'une partie de la population pour un développement ordonné. Quant aux organismes d'aide à l'entreprise, leur fonctionnement ne semble plus adapté à la situation. Il paraît donc utile de proposer ici quelques pistes de réflexion concernant la planification de l'économie régionale, le fonctionnement des organismes d'aide au développement et l'utilisation des TIC.

\section{Réorienter la planification}

Les forces qui impulsent l'activité économique dans la Capitale nationale et dans Chaudière-Appalaches apparaissent plutôt sur la défensive devant l'évolution des aspects majeurs de la conjoncture : accords de libre-échange, force du dollar canadien, mondialisation, vigueur de l'économie asiatique. Les collectivités de nos deux régions accueillent ces changements le plus souvent avec un certain fatalisme, soulignant plutôt leurs aspects négatifs. Il existe pourtant des créneaux avantageux, que nos entreprises devraient chercher avec diligence et investir résolument. Elles doivent cependant reconnaître qu'elles n'arriveront plus à concurrencer les pays émergents sur le plan des salaires ni sur la fabrication d'objets résultant d'un travail routinier, nécessitant peu de créativité mais une abondante main-d'œuvre; elles doivent se démarquer par la qualité, la valeur ajoutée, le contenu en savoir de pointe, l'innovation. Il faut de plus reconsidérer nos stratégies parce que la façon dont elles ont été mises en application jusqu'à maintenant a très peu aidé nos PME à percer le marché international.

Les divergences de vues et d'intérêts entre gens d'affaires de la rive nord et de la rive sud n'ont pas permis, jusqu'à présent, une réelle complémentarité, qui est encore à rechercher. Pour le moment, il ne paraît pas utile de vouloir forcer les choses. Plutôt, les deux régions feraient bien de poursuivre, chacune à son rythme, les objectifs qui lui conviennent, tout en gardant ouverts entre elles les canaux appropriés pour l'information et la consultation, en vue de pouvoir, le cas échéant, s'allier et se concerter pour la réalisation d'un projet particulier.

\section{Une sérieuse remise en question de nos stratégies de développement paraît s'imposer.}

\section{Les PME}

Les divers centres de pouvoir tendent à s'appuyer sur des stratégies qui favorisent surtout les grandes entreprises et adoptent des choix qui, le plus souvent, négligent les PME et leur environnement socio-économique. C'est par exemple le cas lorsqu'on s'entend pour définir les priorités régionales de développement. On choisit des grands projets, qui ont certainement leur place, mais on oublie les petites et moyennes entreprises, éléments pourtant essentiels du tissu socio-économique. Il faut continuer de s'intéresser aux grandes entreprises, tout en veillant à mettre en valeur le potentiel considérable de nos PME et de nos collectivités.

Sans aucun doute, nos PME seraient vivement intéressées par toute stratégie de développement qui leur laisserait entrevoir la possibilité réelle de prospérer. 
Mais elles ne s'impliqueront concrètement dans des réseaux et des organisations que si elles peuvent faire entendre leur voix et si les services offerts leur paraissent adaptés à leurs besoins. Ce dessein se concrétisera si des acteurs éclairés, convaincus de l'importance pour les communautés régionales de se prendre en charge, savent s'investir dans des réseaux et les orienter pour en faire la solide assise économique de leur région. Un tel exercice doit se faire de part et d'autre du fleuve; il préparerait le terrain en vue de préciser les projets sur lesquels il pourrait y avoir concertation, et qui seraient la mise en œuvre de la complémentarité souhaitée. On ajoutera ainsi un supplément d'efficacité et de compétitivité aux PME, mais aussi à l'ensemble de la vie économique.

\section{La Ville de Québec}

À la mairie de Québec, l'intention annoncée par l'actuel conseil municipal de veiller en priorité à la gestion interne de la ville est de bon augure. Les infrastructures seront sans doute améliorées et peut-être aussi les conditions de vie des citoyens. Il faut souhaiter que soit réduite l'influence des réseaux gravitant autour de quelques technocrates de la ville qui, sous la précédente administration, se prenaient pour des ministres lorsqu'il s'agissait de promouvoir ou de représenter la ville à l'étranger, ou de fournir des services à des immigrants souhaitant s'installer dans la région de Québec. Un usage judicieux des outils informatiques réduirait considérablement les dépenses affectées à ce dossier et inciterait ces fonctionnaires à s'occuper de leur vraie mission : servir les citoyens.

\section{Sans aucun doute, nos PME seraient vivement intéressées par toute stratégie de développement qui leur laisserait entrevoir la possibilité réelle de prospérer.}

Dans certains domaines, les champs de compétence et de responsabilité entre la Ville, la Commission de la Capitale et le gouvernement ne sont pas clairs. Par exemple : pour un service quelconque, y a-t-il un guichet clairement identifié ou faut-il parcourir un dédale administratif éprouvant? Les relations internationales relèvent-elles de la Ville ou de la Commission de la Capitale ? À cet égard, nous pensons que la Commission de la Capitale ne devrait pas se limiter à la valorisation du patrimoine régional et à l'embellissement de la ville. Elle devrait plutôt élargir son orientation stratégique et accroître son rôle sur la scène nationale et à l'extérieur, comme elle a fait, par exemple, en appuyant l'Association internationale des études québécoises ${ }^{1}$.

\section{Revoir le fonctionnement des organismes d'aide à l'entreprise}

Le modèle d'appui au développement économique de nos deux régions administratives n'est plus vraiment adapté aux exigences de la conjoncture actuelle. Au cœur de ce modèle, les différents organismes d'aide sont des intermédiaires, des ponts entre les dirigeants de PME et les sources de financement public dont ils peuvent bénéficier. Or, les moyens et les méthodes mis en œuvre par ces intermédiaires conviennent peu à la variété des maillages intersectoriels désormais nécessaires; des maillages qui concourent à la stabilité de microéconomies locales fortes. Les bailleurs de fonds doivent comprendre et faire comprendre aux acteurs locaux et régionaux qu'il est temps de réexaminer ce modèle, de simplifier les ponts, au besoin de les supprimer, car leurs interventions se traduisent trop souvent en lourdeur administrative, en délais tatillons, sources d'intenses frustrations.

Le modèle qui, jusqu'à ce jour, a favorisé le développement économique n'offre plus les stratégies qui répondraient comme il se doit à la mondialisation des marchés et des enjeux économiques. Diverses solutions devraient être étudiées : octroi direct aux entreprises par le ministère concerné, fonction confiée aux MRC (Municipalités régionales de comté), privatisation, etc. Les fonds publics, que les gouvernements d'Ottawa et de Québec consacrent à la gestion et au fonctionnement de ces intermédiaires, devraient servir à des activités pour la mise en valeur des atouts des collectivités locales et régionales auprès d'investisseurs étrangers, et pour encourager nos entrepreneurs à conquérir de nouveaux marchés. En somme, la réforme qui s'impose doit favoriser une structure légère, axée sur le long terme plutôt que sur le dépannage. Elle doit, pour renforcer la capacité concurrentielle de nos PME, viser à stimuler les facteurs de productivité, la formation de la main-d'œuvre et la conquête de marchés extérieurs : " La concurrence est un marathon, pas un sprint!», comme le souligne souvent Michael Porter, ce célèbre professeur de l'université Harvard. 


\section{Promouvoir les outils technologiques}

Particulièrement dans le monde occidental, le développement scientifique et technologique exerce une pression très forte sur les collectivités. À des rythmes variés, celles-ci intègrent le savoir scientifique et apprennent à tirer avantage des infinies possibilités de l'informatique. Chez nous, l'objectif de modernisation de l'État repose en partie sur l'appropriation généralisée des TIC en vue de faciliter l'interaction entre l'appareil gouvernemental et les citoyens et d'améliorer la prestation des services à la population et aux entreprises. C'est donc soutenus par les outils technologiques appropriés qu'on préconise un allégement en même temps qu'un rehaussement de l'efficacité des structures. Il faut maintenant trouver la façon de transposer cette ambition dans la vie socioéconomique de nos régions. C'est, semble-t-il, le moyen le plus apte pour assurer à long terme leur prospérité.

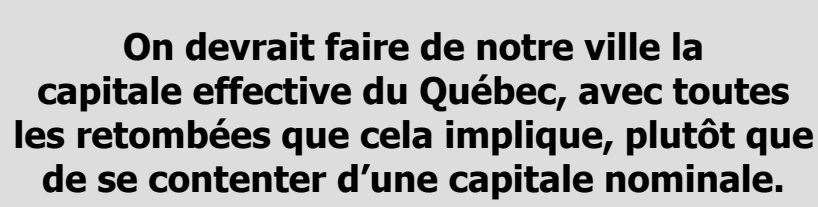

Semblable réorganisation requiert une planification qui devrait réunir tous les principaux intervenants : ministère, MRC, municipalités, acteurs économiques, corps publics, etc. Une collectivité devrait pouvoir s'approprier les outils technologiques qui lui conviennent, les utiliser au soutien de l'activité socio-économique et des services aux individus et aux entreprises; pouvoir de plus effectuer le monitorage de son développement et orienter les initiatives locales en fonction d'une conjoncture mouvante. On ne peut donc envisager une solution toute faite applicable à tous. Il revient donc à chaque collectivité de décider de sa propre réorganisation, selon ses besoins. Il importe par ailleurs, sous prétexte d'éviter une transformation trop brutale, de ne pas plaquer une nouvelle structure sur l'ancienne, et de garder les deux! Se rappeler également que la véritable concurrence, c'est moins entre nos régions qu'avec les économies émergentes.

\section{Conclusion}

En définitive, si certains voient à Québec une « énigme », elle n'est qu'apparente et peut s'expliquer à partir de divers points de vue. L'aspect économique est assurément un élément majeur qu'il importe de réexaminer avec rigueur. Tout d'abord, on devrait faire de notre ville la capitale effective du Québec, avec toutes les retombées que cela implique, plutôt que de se contenter d'une capitale nominale. On devrait renforcer les secteurs médiocres que sont l'accueil des immigrants, le soutien aux PME, la concertation entre acteurs économiques, les transports, etc. La capitale devrait de plus se démarquer par quelques points forts, notamment la culture, le tourisme, les écoles de haut savoir, la recherche, la technologie de pointe et le soutien à l'innovation. Ce sont là quelques champs d'action qui devraient profiter des efforts des élus et des acteurs locaux.

Les comportements et les attitudes de nos élites locales et régionales continueront-ils à assécher le capital de crédibilité que le territoire de Québec détient encore au sein de l'échiquier urbain ? Il est temps de procéder à un aggiornamento de notre modèle économique. Il faut arriver à un changement dans les attitudes et les pratiques des décideurs à divers paliers et chez nos acteurs de développement. Il s'agit de notre capitale, de son rôle ici et dans le monde.

\section{Note}

1 L'Association internationale des études québécoises a pour mission la promotion et le développement des études québécoises au Canada et à l'étranger.

Voir : http://www.aieq.qc.ca/. 